\title{
Finite Size Effects in
}

\section{the Anisotropic $\frac{\lambda}{4 !}\left(\varphi_{1}^{4}+\varphi_{2}^{4}\right)_{d}$ Model}

\author{
C. D. Fosco \\ Centro Atómico Bariloche, 8400 Bariloche, Argentina \\ and \\ N. F. Svaiter \\ Centro Brasileiro de Pesquisas Físicas-CBPF \\ Rua Dr. Xavier Sigaud 150, Rio de Janeiro, RJ, 22290-180, Brazil
}

\begin{abstract}
We consider the $\frac{\lambda}{4 !}\left(\varphi_{1}^{4}+\varphi_{2}^{4}\right)$ model on a d-dimensional Euclidean space, where all but one of the coordinates are unbounded. Translation invariance along the bounded coordinate, $z$, which lies in the interval $[0, L]$, is broken because of the boundary conditions (BC's) chosen for the hyperplanes $z=0$ and $z=L$. Two different possibilities for these BC's boundary conditions are considered: $D D$ and $N N$, where D denotes Dirichlet and $\mathrm{N}$ Newmann, respectively. The renormalization procedure up to one-loop order is applied, obtaining two main results. The first is the fact that the renormalization program requires the introduction of counterterms which are surface interactions. The second one is that the tadpole graphs for $D D$ and $N N$ have the same $z$ dependent part in modulus but with opposite signs. We investigate the relevance of this fact to the elimination of surface divergences.
\end{abstract}




\section{Introduction}

In this paper we consider an interacting quantum field theory model in the presence of boundaries. We shall assume that the system is finite along one dimension $z \in[0, L]$, and infinitely extended along the remaining $(d-1)$ directions.

The presence of geometric restrictions on the domain of one of the coordinates of the system, demands the introduction of classical boundary conditions, to be satisfied by the fields on the two hypersurfaces at $z=0$ and $z=L$. If we restrict ourselves to a real scalar field, Hermiticity of the Hamiltonian leads us to five different (inequivalent) choices for the BC's, namely: $D D$, $N N, D N$, periodic and anti-periodic. The last two choices are usual in the finite-temperature literature, and shall not be dealt with here, since they don't break translation invariance, which is the phenomenon we are concerned with.

Physical systems will be, in general, finite along several directions. For the sake of simplicity we will consider a d-dimensional layered geometry. Although the highly idealized case of planar boundaries misses a whole series of features that are present in the general, curved boundary case, for more general shapes the multiple reflection method can be used to find the correlation functions of the model [1].

Most of the papers in the literature deal with periodic or anti-periodic boundary conditions, where translation symmetry is maintained, and surface effects avoided. Moreover, in quantum systems where translation symmetry is broken, the renormalization procedure is more involved than for translation invariant systems, either bounded or unbounded. 
The diagrammatic expansion and the renormalization program for an unbounded system is conveniently performed in momentum space. On the other hand, when $D D$ or $N N$ BC's are implemented, one may still work with Green's functions at fixed $(d-1)$ dimensional momenta, since there is translation symmetry along those dimensions. As discussed by many authors, associated with the breaking of translational invariance, a new feature emerges: the existence of one particle reducible primitively divergent diagrams. For example, the bare two-point function $G_{0}\left(x, x^{\prime}\right)$ of the scalar model with zero, one or two points on the surfaces have different renormalization constants, respectively.

In this paper we will consider a scalar theory subject to two different classical BC's: $D D$ and $N N$. Besides the lack of translational invariance, we shall face the problem of surface divergences. One way to avoid them is to smooth out the plates surface. But in this case an ambiguity appears, since loop-graphs will depend on an ad-hoc model assumption, namely, the particular features of the smooth walls. Consequently, we prefer to maintain the hard walls assumption. In the context of the Casimir energy of minimally coupled scalar fields, many authors used soft, hard and semihard BC's [2]. Different questions sometimes require more complicated BC's, like the quantum mechanical treatment of the boundary conditions presented by Ford and Svaiter [3], a device implemented to solve a long standing paradox concerning the renormalized energy of minimally and conformally coupled scalar fields.

Besides the above mentioned effects due to the existence of surfaces and the breaking of translation symmetry, we do also have, of course, finite size effects, which are of a different nature. 
Various investigations have been made on this subject, mostly from the quantum statistical mechanics point of view, and we present a short review here. Pathria and co-workers studied the Bose-Einstein condensation of an ideal relativistic Bose-gas confined to a rectangular box of sizes $L_{1}, L_{2}$ and $L_{3}$ with periodic boundary conditions on all the walls [4]. A systematic study of finite size systems and phase transitions was developed by Brezin and Zinn-Justin [5]. These authors studied the $O(N)$ model in two different geometries: the periodic cube and the cylinder along one dimension (the time) and finite and periodic in the $(d-1)$ remaining dimensions. Nemirovsky and Freed considered the same model but in a 'complementary' situation regarding the boundary conditions, namely, $(d-1)$ dimensions are unbounded, and periodicity along the only finite dimension, and [6]. Afterwards, Singh and Pathria studied the $O(N)_{d}$ model confined to geometries with periodic boundary conditions in all directions [7]. The same model in the presence of one mirror localized at $z=0$ was also studied by many authors 8$]$.

Another ingredient, important in finite size systems at criticality, is the concept of finite size scaling. Let us consider a finite system of linear size $L$ and suppose a thermodynamic quantity $P_{L}(t)$ (where $t$ is the reduced temperature) becomes singular as $t \rightarrow 0$. Defining $\frac{P_{L}(t)}{P(t)}=g(L, t)$, where $P(t)$ is the bulk value of $P_{L}(t)$, the statement of finite size scaling is that $g(L, t)=f(L / \xi(t))$, where $\xi(t)$ is the correlation length. In other words, finite size scaling predicts that, for large $L$, the dependence on $L$ of the singular contributions to thermodynamics functions scales with the correlation length, and is described by universal scaling functions. It may be pointed out that finite size systems must be classified in two distinct groups with respect to finite size scaling, 
depending on whether the B.C.'s break translation invariance or not. For finite size systems where translation invariance is maintained (for example, a periodic cube, or a cylinder infinite along one dimension, and finite and periodic in the $(d-1)$ other dimensions), finite size scaling is easily understood.

The renormalization group equations are insensitive to finite size effects (since the renormalization is related to short distance singularities) and must, accordingly, be maintained in such finite size geometries. However, the solutions to those equations must be different from those for the unconstrained systems, because correlation functions can depend on the additional dimensional quantities (the lengths of the compactified dimensions) and finite size scaling is present.

For the cases of $D D, N N$ or $D N$ B.C.'s, the situation is quite different, since it is much more difficult to decide if a given interaction is relevant, irrelevant or marginal, the reason being that the propagator of the critical theory satisfies B.C.'s which can interfere with the power counting.

Finite size effects have also been extensively studied in the quantum field theory context during the last twenty years. In flat spacetime with one compactified dimension, the mass can depend upon the periodicity length [9]. This phenomenon is of particular interest in theories with broken symmetry, as it allows topological effects to play a role in the restoration of symmetry. An equivalent mechanism is at work when we assume that the fields are in thermal equilibrium with a reservoir at temperature $\beta^{-1}[10$. Finite size effects in quantum field theory with periodic boundary conditions in the spatial section at finite temperature was analyzed by many authors. See, for example, [1], and references therein. 
In the context of non-Abelian gauge field theories at zero temperature, cavity QCD was studied by many authors [12]. Hanson and Jaffe and also Hanson, Jonhson and Peterson dealt with quantum fields in bounded domains with broken translation invariance. Regarding Abelian gauge theories, quantum electrodynamics in the presence of conducting plates has also been the focus of research [13]. Based on the fact that the B.C.'s for the electron field could lead to additional contributions to the Casimir force, Bordag et al. and also Robaschik et al. adopted the following model. A photon field obeys classical B.C.'s on perfectly conducting plates, while the B.C's for the fermion field are free. These authors assumed that the electromagnetic field also exists in the region outside the plates (two simple connected domains). Chodos and Thorn investigated the self-energy of fermions used different B.C's (the slab-bag), where the fermionic field is confined between two parallel plates and the photon field is unconfined. In flat spacetime, for systems where some dimensions are compactified but translational invariance is maintained, Toms [14] and also Birrel and Ford [15] have shown that all the counterterms are independent on the compactified spatial size. A more general discussion has been given by Banach [16]. This author proved that a topological identification (periodic or anti-periodic B.C.'s) does not introduce new counterterms into the theory. As stressed by many authors, were this not the case there would be a catastrophe in the renormalizability of the model.

For translation invariant systems, because of Poincaré invariance, one should expect that overlapping divergences will not obstruct the implementation of the renormalization program [17]. In systems where Poincaré invariance does not hold, these proofs do not apply, and one must show 
that it is still possible to implement such program. A technical difficulty is also met here, since the presence of geometric restrictions makes Feynman diagrams harder to compute than is ordinary quantum field theory in unbounded systems. An crucial work on this subject has been presented by Symanzik [18]. Of particular importance are also the papers by Nemirovsky and Freed, and Krech and Dietrich [19].

In this work we shall consider an anisotropic scalar model, in a d-dimensional Euclidean space, where the first $(d-1)$ coordinates are unbounded and the last one lies in the interval $[0, L]$. We analyze two different translation symmetry breaking B.C.'s: $D D$ and $N N$ on the plates. We first present a rederivation of the fact that to renormalize the theory one has to introduce counterterms as surface interactions. We also show that the tadpole graphs for $D D$ and for $N N$ B.C.'s have the same modulus for their $z$-dependent parts, but their signs are opposite. We study the possible use of this property to get rid of the surface divergences.

The organization of the paper is as follows: In section II we present the general formalism. In section III we discuss the slab configurations, dealing with the two-point and four-point functions, both for $D D$ and $N N$ b.c. In section IV we analyze the divergences of the translational invariant part of the tadpoles. Section V deals with the analysis of the ultraviolet and infrared divergences of the $z$-dependent part of the tadpoles. Finally, section VI contains our conclusions. Throughout this paper we use $\hbar=c=1$. 


\section{General formalism and the scalar anisotropic model}

Let us consider $Z[J]$, the generating functional of complete Green's functions for a scalar field in a $d$-dimensional Euclidean space

$$
Z[J]=\int \mathcal{D} \varphi e^{-S[\varphi]+\int J(x) \varphi(x)}
$$

where

$$
S[\varphi]=\int d^{d} x[\mathcal{L}(\varphi(x), \partial \varphi(x))]
$$

$\mathcal{D} \varphi$ is the appropriate measure, and $S[\varphi]$ is the classical action associated with the scalar field. The quantity $Z[J]$ can be regarded as a functional integral representation for the imaginary time evolution operator $\left\langle\varphi_{2}\left|U\left(t_{2}, t_{1}\right)\right| \varphi_{1}\right\rangle$ with the boundary conditions: $\varphi\left(t_{1}, x\right)=\varphi_{1}(\vec{x})$ and $\varphi\left(t_{2}, x\right)=$ $\varphi_{2}(\vec{x})$. The quantity $Z[J]$ gives the transition amplitude from the initial state $\left|\varphi_{1}\right\rangle$ to the final state $\left|\varphi_{2}\right\rangle$ in the presence of some scalar source $J(x)$, of compact support. Regarding the Lagrangian density $\mathcal{L}$, we shall assume it to be

$$
\mathcal{L}(\varphi)=\frac{1}{2}(\partial \varphi)^{2}+\frac{1}{2} m^{2} \varphi^{2}+\frac{1}{4 !} \lambda \varphi^{4}
$$

The n-point correlation functions are given by the expectation value with respect to the weight $e^{-S(\varphi)}$ defined as

$$
G^{(n)}\left(x_{1}, x_{2}, . ., x_{n}\right)=<\varphi\left(x_{1}\right) \ldots \varphi\left(x_{n}\right)>=\left.\frac{1}{Z(J)} \frac{\delta^{n} Z(J)}{\delta J\left(x_{1}\right) \delta J\left(x_{2}\right) . . \delta J\left(x_{n}\right)}\right|_{J=0}
$$




$$
=\int \mathcal{D} \varphi \varphi\left(x_{1}\right) \ldots \varphi\left(x_{n}\right) e^{-S[\varphi]} .
$$

As usual, $W(J)$, the generating functional for connected correlation functions of the elementary fields shall be given by $W(J)=\ln Z(J)$. Thus

$$
G_{c}^{(n)}\left(x_{1}, x_{2}, . ., x_{n}\right)=\left.\frac{\delta^{n} W(J)}{\delta J\left(x_{1}\right) \delta J\left(x_{2}\right) . . \delta J\left(x_{n}\right)}\right|_{J=0}=<\varphi\left(x_{1}\right) \ldots \varphi\left(x_{n}\right)>_{c} .
$$

Finally, $\Gamma\left(\varphi_{0}\right)$, the generating functional of connected one-particle irreducible correlation functions is introduced by performing a Legendre transformation on $W[J]$,

$$
\Gamma\left(\varphi_{0}\right)=-W(J)+\int d^{d} x \varphi(x) J(x)
$$

and

$$
\Gamma^{(n)}\left(x_{1}, x_{2}, . ., x_{n}\right)=\left.\frac{\delta^{n} \Gamma\left(\varphi_{0}\right)}{\delta \varphi_{0}\left(x_{1}\right) \delta \varphi_{0}\left(x_{2}\right) . . \delta \varphi_{0}\left(x_{n}\right)}\right|_{\varphi_{0}=0}
$$

where

$$
\varphi_{0}(x)=\frac{\delta W}{\delta J(x)} .
$$

If $\lambda=0$ the partition function $Z(J)$ can be calculated exactly i.e.

$$
Z_{0}(J)=\exp \left(\frac{1}{2} \int d^{d} x d^{d} y J(x) D\left(x-y, m^{2}\right) J(y)\right)
$$

where

$$
\left(-\Delta_{x}+m^{2}\right) D\left(x-y, m^{2}\right)=\delta^{d}(x-y) .
$$


For $\lambda \neq 0$ it is not possible to find exactly $Z(J)$ and perturbation theory is mandatory. This expansion stems from the formal identity:

$$
Z(J)=\exp \left[-\frac{\lambda}{4 !} \int d^{d} x\left(\frac{\delta}{\delta J(x)}\right)^{4}\right] Z_{0}(J)
$$

From now on, we shall consider a generalization of the previous case, namely, the anisotropic Landau-Ginzburg model for a $N=2$ component order parameter with a Lagrange density $\mathcal{L}=$ $\mathcal{L}_{0}+\mathcal{L}_{\text {int }}$, where

$$
\mathcal{L}_{0}\left(\varphi_{1}, \varphi_{2}\right)=\frac{1}{2}\left(\partial \varphi_{1}\right)^{2}+\frac{1}{2}\left(\partial \varphi_{2}\right)^{2}+\frac{1}{2} m^{2} \varphi_{1}^{2}+\frac{1}{2} m^{2} \varphi_{2}^{2}
$$

and

$$
\mathcal{L}_{i n t}=\frac{\lambda}{4 !}\left(\varphi_{1}^{4}+\varphi_{2}^{4}\right)
$$

To generate the n-point functions we have to introduce two scalar sources $J_{1}(x)$ and $J_{2}(x)$ coupled linearly with the fields $\varphi_{1}(x)$ and $\varphi_{2}(x)$ respectively. Integrating out the fields, we obtain $Z\left(J_{1}\right)$ and $Z\left(J_{2}\right)$ and the total partition function of the model factorizes: $Z\left(J_{1}, J_{2}\right)=Z\left(J_{1}\right) Z\left(J_{2}\right)$, where

$$
Z\left(J_{1,2}\right)=\frac{1}{N_{1,2}} \exp \left(-\frac{\lambda}{4 !} \int d^{d} x\left(\frac{\delta}{\delta J_{1,2}(x)}\right)^{4}\right) Z_{0}\left(J_{1,2}\right)
$$

and

$$
Z_{0}\left(J_{1,2}\right)=\exp \left(\frac{1}{2} \int d^{d} x d^{d} y J_{1,2}(x) G_{1,2}^{(2)}\left(x-y, m^{2}\right) J_{1,2}(y)\right)
$$

In the above equation, $G_{1}^{(2)}\left(x-y, m^{2}\right)$ and $G_{2}^{(2)}\left(x-y, m^{2}\right)$ are the free propagators, solutions of the inhomogeneous equations $(i=1,2)$.

$$
\left(-\Delta_{x}+m^{2}\right) G_{i}^{(2)}\left(x-y, m^{2}\right)=\delta^{d}(x-y) .
$$


The partition function applies to arbitrary geometries, and classical B.C.'s must be implemented on the Green's functions. As discussed before, we will assume that the system is confined between two parallel plates localized at $z=0$ and $z=L$, and use the Cartesian coordinates $x^{\mu}=(\vec{r}, z)$ where $\vec{r}$ is a $(d-1)$ dimensional vector perpendicular to the $z$ direction. A question that arises in such a model is related to the renormalization conditions. It is well known that for fields interacting with a thermal bath defined in manifolds where the spacelike sections are non-compact the mass and coupling constant counterterms are temperature independent. Using dimensional regularization [20] it was proved that for fields defined on manifolds where the spacelike sections are non-compact, or compact in at least one dimension, but with the other dimensions noncompactified, the mass and coupling constant counterterms are size and temperature independent at the two-loop level. In a perturbative scheme, the renormalized theory is fixed by the renormalization conditions for the superficially divergent vertex functions (the one particle irreducible parts of the connected Green's functions). In other words, in the conventional renormalizable (translational invariant) theory the ultraviolet divergences can be absorbed by counterterms related to the field, mass and coupling constant. A question of fundamental importance is how the renormalization program can be implemented in systems where translational invariance is broken. The purpose of the next section is to analyze this question for the case of the anisotropic model at the one-loop approximation. 


\section{$3 \quad$ Finite size effects and classical boundary conditions}

For the cubic anisotropic model, we define the boundary conditions over the plates for the fields $\varphi_{1}(x)$ and $\varphi_{2}(x)$. For the $\varphi_{1}(x)$ field we assume Dirichlet-Dirichlet boundary conditions i.e:

$$
\left.\varphi_{1}(\vec{r}, z)\right|_{z=0}=\left.\varphi_{1}(\vec{r}, z)\right|_{z=L}=0
$$

and for the $\varphi_{2}(x)$ we will assume Newmann-Newmann boundary conditions, i.e.:

$$
\left.\frac{\partial}{\partial z} \varphi_{2}(\vec{r}, z)\right|_{z=0}=\left.\frac{\partial}{\partial z} \varphi_{2}(\vec{r}, z)\right|_{z=L}=0
$$

It is well known that ${ }^{4} \mathrm{He}$ films close to the $\lambda$ transition satisfies $D D$ b.c. Another well known example of such kind of boundary conditions is the electromagnetic field. It was shown that for an electromagnetic field confined in a perfectly conducting cavity, it is possible to treat the electric and magnetic modes separately, where each one satisfies Dirichlet and Newmann B.C.'s, respectively [21]. Going back to our discussion, since the translational invariance is not preserved, let us use a Fourier expansion of the fields in the following form:

$$
\varphi(\vec{r}, z)=\frac{1}{(2 \pi)^{\frac{d-1}{2}}} \int d^{d-1} p \sum_{n} \phi_{n}(\vec{p}) e^{i \vec{p} \cdot \vec{r}} u_{n}(z) .
$$

where the $u_{n}(z)$ are the normalized eigenfunctions of the operator $-\frac{d^{2}}{d z^{2}}$ satisfying the completeness and orthonormality relations, i.e.,

$$
\sum_{n} u_{n}(z) u_{n}^{*}\left(z^{\prime}\right)=\delta\left(z-z^{\prime}\right)
$$




$$
\int_{0}^{L} d z u_{n}(z) u_{n^{\prime}}^{*}(z)=\delta_{n, n^{\prime}}
$$

and finally

$$
-\frac{d}{d z^{2}} u_{n}(z)=k_{n}^{2} u_{n}(z)
$$

where $k_{n}=\frac{n \pi}{L}, n=1,2$. for $D D$ b.c and $n=0,1,2$. for $N N$ b.c.

It should be noted that this kind of expansion in an orthonormal set corresponding to the eigenfunctions of the Hermitian operator $-\frac{d^{2}}{d z^{2}}$ defined on a finite interval could be quite straightforwardly generalized to different anisotropic models. For example, we might consider a scalar field defined on Euclidean space with all the coordinates unbounded, but with a mass having an anisotropy along the $z$ coordinate. Namely, the Lagrangian density could be

$$
\mathcal{L}=\frac{1}{2}(\partial \varphi)^{2}+\frac{1}{2}\left(m^{2}+\mu^{2}(z)\right) \varphi^{2}+\frac{\lambda}{4 !} \varphi^{4}
$$

which is, of course, non invariant under translations in $z$, except for the trivial case of a constant $\mu(z)$. If now we assume the $u_{n}(z)$ 's to denote the normalized eigenfunctions of the Hermitian operator

$$
h=-\frac{d^{2}}{d z^{2}}+\mu(z)
$$

with

$$
h u_{n}(z)=\lambda_{n}^{2} u_{n}(z)
$$

the expansion (19) still holds. We assume $\mu$ to be a non negative function, so that $h$ is definite positive. 
We see that the case of Dirichlet B.C.'s could be obtained starting from an unbounded $z$ coordinate, and using the anisotropic mass $\mu(z)=\mu\left(\frac{z}{L}\right)^{n}$, with $n \rightarrow \infty$, and $\mu$ a (positive) constant. For a study of Dirac fermions with a space dependent mass see for example ref. [22].

Coming back to the case of $D D$ and $N N$ boundary conditions, the eigenfunctions are, respectively,

$$
u_{n}(z)=\sqrt{\frac{2}{L}} \sin \left(\frac{n \pi z}{L}\right) n=1,2 . .
$$

and

$$
u_{n}(z)=\sqrt{\frac{2}{L}} \cos \left(\frac{n \pi z}{L}\right) n=1,2, \cdots
$$

For $N N$ B.C.'s, we also have the zero mode $u_{0}(z)=\frac{1}{\sqrt{L}}$. The free propagator can be expressed in the following form:

$$
G_{0}^{(2)}\left(\vec{r}, z, z^{\prime}\right)=\frac{1}{(2 \pi)^{d-1}} \int d^{d-1} p \sum_{n} e^{i \vec{p} \cdot \vec{r}} u_{n}(z) u_{n}^{*}\left(z^{\prime}\right) G_{0, n}(\vec{p})
$$

where is not difficult to show that $G_{0, n}(\vec{p})$ is given by

$$
G_{0, n}(\vec{p})=\left(\vec{p}^{2}+k_{n}^{2}+m^{2}\right)^{-1}
$$

For the anisotropic mass case, we would have instead:

$$
G_{0, n}(\vec{p})=\left(\vec{p}^{2}+\lambda_{n}^{2}+m^{2}\right)^{-1}
$$

As we discussed before, for translational invariant systems we have $G_{0}^{(2)}\left(x, x^{\prime}\right)=G_{0}^{(2)}\left(x-x^{\prime}\right)$ and from coordinate space Feynman rules we can go to momentum space representation, which is 
the more convenient framework to analyze the divergences of the theory. Translation invariance is reflected in momentum conservation conditions. Since our system possesses translation invariance along the direction parallel to the plates, the parallel momentum, $\vec{p}$, is conserved. In this case a convenient representation is a mixed $(\vec{p}, z)$ space. The Feynman rules for different boundary conditions was derived in many references and we will not repeat the rules here. For a careful study of Feynman rules in such systems see for example Ref. [23]. Let us study the one-loop correction to the bare two-point function $G_{0}^{(2)}\left(x, x^{\prime}\right)$, both for the $D D$ and $N N$ cases. Using the Feynman rules (see fig.(1)) we have:

$$
G_{0}^{(2)}\left(\lambda, \vec{r}_{1}, z_{1}, \vec{r}_{3}, z_{3}\right)=\frac{\lambda}{2} \int d^{d-1} r_{2} \int_{0}^{L} d z_{2} G_{0}^{(2)}\left(\vec{r}_{1}, z_{1} ; \vec{r}_{2}, z_{2}\right) G_{0}^{(2)}\left(\vec{r}_{2}, z_{2} ; \vec{r}_{2}, z_{2}\right) G_{0}^{(2)}\left(\vec{r}_{2}, z_{2} ; \vec{r}_{3}, z_{3}\right)
$$

or

$$
G_{0}^{(2)}\left(\lambda, \vec{r}_{1}, z_{1}, \vec{r}_{3}, z_{3}\right)=\frac{\lambda}{2} \int d^{d-1} r_{2} \int_{0}^{L} d z_{2} G_{0}^{(2)}\left(\vec{r}_{1}-\vec{r}_{2} ; z_{1}, z_{2}\right) G_{0}^{(2)}\left(\overrightarrow{0}, z_{2}\right) G_{0}^{(2)}\left(\vec{r}_{2}-\vec{r}_{3} ; z_{2}, z_{3}\right) .
$$

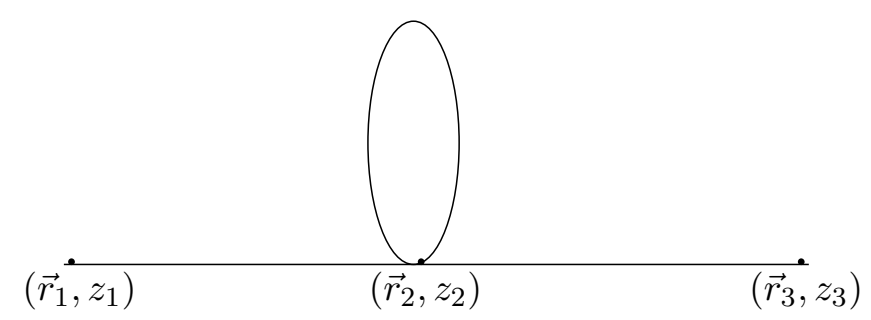

Figure 1: The two point function for $\varphi_{1}(x)$ 
Each of these expressions can, for the case of $D D$ B.C.'s, be expanded as

$$
\begin{aligned}
& G_{0}^{(2)}\left(\vec{r}_{1}-\vec{r}_{2}, z_{1}, z_{2}\right)=\frac{2}{L} \frac{1}{(2 \pi)^{d-1}} \sum_{n=1}^{\infty} \sin \left(\frac{n \pi z_{1}}{L}\right) \sin \left(\frac{n \pi z_{2}}{L}\right) \int d^{d-1} p \frac{e^{i \vec{p} \cdot\left(\vec{r}_{1}-\vec{r}_{2}\right)}}{\left(\vec{p}^{2}+\left(\frac{n \pi}{L}\right)^{2}+m^{2}\right)} . \\
& G_{0}^{(2)}\left(\vec{r}_{2}-\vec{r}_{3}, z_{2}, z_{3}\right)=\frac{2}{L} \frac{1}{(2 \pi)^{d-1}} \sum_{n^{\prime}=1}^{\infty} \sin \left(\frac{n \pi z_{2}}{L}\right) \sin \left(\frac{n \pi z_{3}}{L}\right) \int d^{d-1} p \frac{e^{i \vec{p} \cdot\left(\vec{r}_{2}-\vec{r}_{3}\right)}}{\left(\vec{p}^{2}+\left(\frac{n^{\prime} \pi}{L}\right)^{2}+m^{2}\right)}
\end{aligned}
$$

and finally

$$
G_{0}^{(2)}\left(\overrightarrow{0}, z_{2}\right)=\frac{2}{L} \frac{1}{(2 \pi)^{d-1}} \sum_{n^{\prime \prime}=1}^{\infty} \sin ^{2} \frac{n^{\prime \prime} \pi z_{2}}{L} \int d^{d-1} p \frac{1}{\left(\vec{p}^{2}+\left(\frac{n^{\prime \prime} \pi}{L}\right)^{2}+m^{2}\right)} .
$$

Although the functions $G_{0}^{(2)}\left(\vec{r}_{1}-\vec{r}_{2}, z_{1}, z_{2}\right)$ and $G_{0}^{(2)}\left(\vec{r}_{2}-\vec{r}_{3}, z_{2}, z_{3}\right)$ are singular at $\vec{r}_{1}=\vec{r}_{2}, z_{1}=z_{2}$ and $\vec{r}_{2}=\vec{r}_{3}, z_{2}=z_{3}$, the singularities are integrable (for points outside the plates), consequently only the tadpole is divergent and needs a regularization and renormalization procedure. A straightforward calculation yields the order $\lambda$ correction to the bare two-point function in the one-loop approximation :

$$
\begin{aligned}
G_{0}^{(2)}\left(\lambda, \vec{r}_{1}-\vec{r}_{3}, z_{1}, z_{3}\right)= & \frac{2}{L^{2}} \frac{1}{(2 \pi)^{d-1}} \int_{0}^{L} d z_{2} \sum_{n, n^{\prime}=1}^{\infty} \sin \left(\frac{n \pi z_{1}}{L}\right) \sin \left(\frac{n \pi z_{2}}{L}\right) \sin \left(\frac{n^{\prime} \pi z_{2}}{L}\right) \sin \left(\frac{n^{\prime} \pi z_{3}}{L}\right) \\
& \int d^{d-1} p \frac{e^{i \vec{p}\left(\vec{r}_{1}-\vec{r}_{3}\right)}}{\left(\vec{p}^{2}+\left(\frac{n \pi}{L}\right)^{2}+m^{2}\right)\left(\vec{p}^{2}+\left(\frac{n^{\prime} \pi}{L}\right)^{2}+m^{2}\right)} T_{D D}\left(L, m, d, z_{2}\right)
\end{aligned}
$$

where, since we will use dimensional regularization, we introduce a dimensional parameter $\mu$, and define $g=\lambda \mu^{4-d}$. The expression for the tadpole $T_{D D}(L, m, d, z)$ is then:

$$
T_{D D}(L, m, d, z)=\frac{2 g}{L} \frac{1}{(2 \pi)^{d-1}} \sum_{n=1}^{\infty} \sin ^{2}\left(\frac{n \pi z}{L}\right) \int d^{d-1} p \frac{1}{\left(\vec{p}^{2}+\left(\frac{n \pi}{L}\right)^{2}+m^{2}\right)}
$$

The tadpole graph in the case of N-N B.C.'s can be also easily found, and it is given by

$$
T_{N N}(L, m, d, z)=\frac{g}{L} \frac{1}{(2 \pi)^{d-1}} \int d^{d-1} k \frac{1}{\left(\vec{k}^{2}+m^{2}\right)}
$$




$$
+\frac{2 g}{L} \frac{1}{(2 \pi)^{d-1}} \sum_{n=1}^{\infty} \cos ^{2}\left(\frac{n \pi z}{L}\right) \int d^{d-1} p \frac{1}{\left(\vec{p}^{2}+\left(\frac{n \pi}{L}\right)^{2}+m^{2}\right)} .
$$

Note that both $T_{D D}(L, m, d, z)$ and $T_{N N}(L, m, d, z)$ diverge in their continuum momenta integrals and also in the $n$ summation. In the next section we will analyze the ultraviolet behaviour of the bare two-point functions i.e $T_{D D}(L, m, d, z)$ and $T_{N N}(L, m, d, z)$. Before dealing with the renormalization of the one-loop two point function, let us, by the sake of completeness, discuss the bare four-point function. The expression for the bare four-point function is given below, and the same analysis of the divergences can be done. In this paper we will not implement the renormalization program of the four point-function (which follows using the same procedure used in the two-point function). Our object of interest is the two-point function, since it is the fundamental quantity that measures the vacuum activity. Using the Feynman rules, $G_{0}^{(4)}\left(\lambda, x_{1}, x_{2}, x_{3}, x_{4}\right)$, the order $\lambda^{2}$ correction to the bare four-point function, is given by

$$
\begin{aligned}
& G_{0}^{(4)}\left(\lambda, \vec{r}_{1}, z_{1}, \vec{r}_{2}, z_{2}, \vec{r}_{5}, z_{5}, \vec{r}_{6}, z_{6}\right)=\frac{1}{2} \int d^{d-1} r_{3} \int d^{d-1} r_{4} \int_{0}^{L} d z_{3} \int_{0}^{L} d z_{4} G_{0}^{(2)}\left(\vec{r}_{1}-\vec{r}_{3}, z_{1}, z_{3}\right) \\
& G_{0}^{(2)}\left(\vec{r}_{2}-\vec{r}_{3}, z_{2}, z_{3}\right)\left[G_{0}^{(2)}\left(\vec{r}_{3}-\vec{r}_{4}, z_{3}, z_{4}\right)\right]^{2} G_{0}^{(2)}\left(\vec{r}_{4}-\vec{r}_{5}, z_{4}, z_{5}\right) G_{0}^{(2)}\left(\vec{r}_{4}-\vec{r}_{6}, z_{4}, z_{6}\right) .
\end{aligned}
$$

Again, all $G_{0}$ 's are singular at the same points, but the singularities are integrable, except for $G_{0}^{(2)}\left(\vec{r}_{3}, z_{3}, \vec{r}_{4}, z_{4}\right)$, consequently, to renormalize the bare four-point function we have the regularize the 1 PI four-point function $\Gamma^{(4)}\left(\lambda, \vec{r}_{3}-\vec{r}_{4}, z_{3}, z_{4}\right)=\left[G_{0}^{(2)}\left(\lambda, \vec{r}_{3}-\vec{r}_{4}, z_{3}, z_{4}\right)\right]^{2}$, which, for DD B.C.'s, is given by

$$
G_{0}^{(2)}\left(\vec{r}_{3}-\vec{r}_{4}, z_{3}, z_{4}\right)=\frac{2 g}{L} \frac{1}{(2 \pi)^{d-1}} \sum_{n=1}^{\infty} \sin \left(\frac{n \pi z_{3}}{L}\right) \sin \left(\frac{n \pi z_{4}}{L}\right) \int d^{d-1} p \frac{e^{i \vec{p} \cdot\left(\vec{r}_{3}-\vec{r}_{4}\right)}}{\left(\vec{p}^{2}+\left(\frac{n \pi}{L}\right)^{2}+m^{2}\right)}
$$




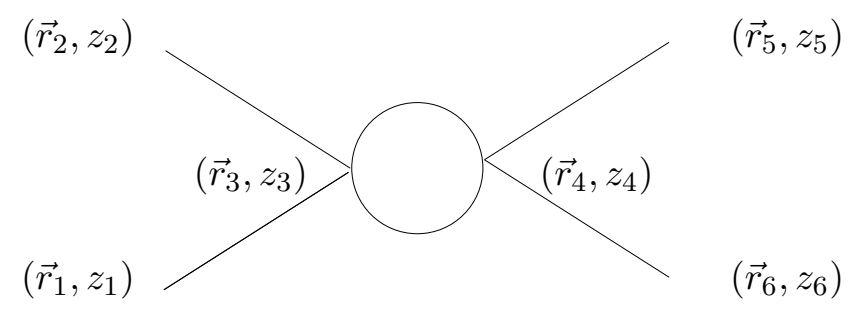

Figure 2: The four point function for $\varphi_{1}(x)$

A convenient way to express $G_{0}\left(\vec{r}_{3}-\vec{r}_{4}, z_{3}, z_{4}\right)$ is the following. Let us define $\vec{\rho}=\vec{r}_{3}-\vec{r}_{4}$, and also $z_{3}-z_{4}=u, z_{3}+z_{4}=v$ then it is possible to write

$$
G_{0}^{(2)}\left(\vec{r}_{3}-\vec{r}_{4}, z_{3}, z_{4}\right)=G_{0}^{(2)}(\vec{\rho}, u)+G_{0}^{(2)}(\vec{\rho}, v),
$$

where

$$
G_{0}^{(2)}(\vec{\rho}, u)=\frac{g}{L} \frac{1}{(2 \pi)^{d-1}} \sum_{n=1}^{\infty} \cos \left(\frac{n \pi u}{L}\right) \int d^{d-1} p \frac{e^{i \vec{p} \cdot \vec{\rho}}}{\left(\vec{p}^{2}+\left(\frac{n \pi}{L}\right)^{2}+m^{2}\right)},
$$

and also

$$
G_{0}^{(2)}(\vec{\rho}, v)=-\frac{g}{L} \frac{1}{(2 \pi)^{d-1}} \sum_{n=1}^{\infty} \cos \left(\frac{n \pi v}{L}\right) \int d^{d-1} p \frac{e^{i \vec{p} \cdot \vec{\rho}}}{\left(\vec{p}^{2}+\left(\frac{n \pi}{L}\right)^{2}+m^{2}\right)} .
$$

For simplicity let us choose $m=0$ and $\vec{\rho}=0$. Using the fact that $d^{d-1} p=p^{d-2} d p d \Omega_{d-1}$ and $\int d \Omega_{d-1}=\frac{2 \pi \frac{d-1}{2}}{\Gamma\left(\frac{d-1}{2}\right)}$, a straightforward calculation yields

$$
\left.G_{0}^{(2)}(\vec{\rho}, u, v)\right|_{\rho=0}=B_{1}(d, L, u)+B_{2}(d, L, v)+B_{3}(d, L, v)+B_{4}(d, L, u),
$$

where

$$
B_{1}(d, L, u)=\frac{2 g}{L} h_{2}(d) \int d k k^{d-3} \operatorname{coth} L k \cosh k u .
$$




$$
\begin{gathered}
B_{2}(d, L, v)=-\frac{2 g}{L} h_{2}(d) \int d k k^{d-3} \operatorname{coth} k L \cosh k v . \\
B_{3}(d, L, v)=\frac{2 g}{L} h_{2}(d) \int d k k^{d-3} \sinh k v
\end{gathered}
$$

and finally

$$
B_{4}(d, L, u)=-\frac{2 g}{L} h_{2}(d) \int d k k^{d-3} \sinh k u \text {. }
$$

It is worth mentioning that the structure of the divergences of Eqs.(45.48) are the same as for the tadpoles, as we will see. In the next section we will analyze the renormalization program for the two-point functions in both cases of $D D$ and $N N$ boundary conditions.

\section{Analysis of the ultraviolet divergences of $T_{D D}(L, m, d, z)$ and $T_{N N}(L, m, d, z)$}

The aim of this section is to analyze the structure of the divergences of the bare two-point functions for both cases $D D$ and $N N$ boundary conditions. Let us start from the expression of the vacuum activity for the case of $D D$ boundary conditions, i.e.,

$$
T_{D D}(L, m, d, z)=\frac{2 g}{L} \frac{1}{(2 \pi)^{d-1}} \sum_{n=1}^{\infty} \sin ^{2}\left(\frac{n \pi z}{L}\right) \int d^{d-1} p \frac{1}{\left(\vec{p}^{2}+\left(\frac{n \pi}{L}\right)^{2}+m^{2}\right)}
$$

Using trigonometric identities and also the relation [24]

$$
\sum_{n=1}^{\infty} \frac{\cos n x}{n^{2}+a^{2}}=-\frac{1}{2 a^{2}}+\frac{\pi}{2 a} \frac{\cosh a(\pi-x)}{\sinh \pi a}
$$


which is valid for $0 \leq x \leq 2 \pi$, it is easy to show that the vacuum activity in the case of $D D$ b.c. is given by

$$
T_{D D}(L, m, d, z)=\frac{g}{2 L} \frac{1}{(2 \pi)^{d-1}} \sum_{n=-\infty}^{\infty} \int d^{d-1} p \frac{1}{\left(\vec{p}^{2}+\left(\frac{n \pi}{L}\right)^{2}+m^{2}\right)}-g f_{2}(d, L, m, z)
$$

where

$$
f_{2}(L, m, d, z)=\frac{1}{2} \frac{1}{(2 \pi)^{d-1}} \int d^{d-1} p \frac{1}{\left(\vec{p}^{2}+m^{2}\right)^{\frac{1}{2}}} \frac{\cosh \left((L-2 z)\left(\vec{p}^{2}+m^{2}\right)^{\frac{1}{2}}\right)}{\sinh \left(L\left(\vec{p}^{2}+m^{2}\right)^{\frac{1}{2}}\right)} .
$$

In an analogous way, it is also possible to calculate the vacuum activity for the $N N$ b.c. i.e. $T_{N N}(L, m, d, z)$ and we obtain

$$
T_{N N}(L, m, d, z)=\frac{g}{2 L} \frac{1}{(2 \pi)^{d-1}} \sum_{n=-\infty}^{\infty} \int d^{d-1} p \frac{1}{\left(\vec{p}^{2}+\left(\frac{n \pi}{L}\right)^{2}+m^{2}\right)}+g f_{2}(L, m, d, z)
$$

Since $T_{D D}(L, m, d, z)$ and $T_{N N}(L, m, d, z)$ have the same functional form, both have the same kind of ultraviolet divergences. Let us define $f_{1}(L, m, d)$ by:

$$
f_{1}(L, m, d)=\frac{1}{2 L} \frac{1}{(2 \pi)^{d-1}} \sum_{n=-\infty}^{\infty} \int d^{d-1} p \frac{1}{\left(\vec{p}^{2}+\left(\frac{n \pi}{L}\right)^{2}+m^{2}\right)}
$$

The equation above has ultraviolet divergences, but it is (formally) proportional to the tadpole in finite temperature field theory, after the identification: $\beta \equiv 2 L$. To deal with the divergences of the one-loop two-point function at finite temperature we have to do frequency sums and $(d-$ 1) dimensional integrals for the continuum momenta. The most popular method to deal with Matsubara sums is to analytic extension away from the discrete complex energies down to real axis with the replacement of the energy sums by contour integrals [25]. We prefer to use dimensional regularization in the continuum [20], and afterwards to analytically extend the modified Epstein 
zeta function which appear after the dimensional regularization [26]. Since the formalism has already been developed by Malbouisson and Svaiter in [27], we will only sketch the procedure here. First we have to use a well known result of dimensional regularization, i.e.

$$
\int \frac{d^{d} k}{\left(k^{2}+a^{2}\right)^{s}}=\frac{\pi^{\frac{d}{2}}}{\Gamma(s)} \Gamma\left(s-\frac{d}{2}\right) \frac{1}{a^{2 s-d}}
$$

and let us define the modified Epstein zeta function $\zeta(z, a)$ by:

$$
\zeta(z, a)=\sum_{n=-\infty}^{\infty} \frac{1}{\left(n^{2}+a^{2}\right)^{z}} \quad a^{2}>0
$$

which is analytic for $\operatorname{Re}(z)>\frac{1}{2}$. It is possible to analytic extend the modified Epstein zeta function where the integral representation is valid for $\operatorname{Re}(z)<1$, [28]:

$$
\sum_{n=-\infty}^{\infty}\left(n^{2}+a^{2}\right)^{-z}=a^{1-2 z}\left[\sqrt{\pi} \frac{\Gamma\left(z-\frac{1}{2}\right)}{\Gamma(z)}+4 \sin \pi z \int_{1}^{\infty} \frac{\left(t^{2}-1\right)^{-z} d t}{\mathrm{e}^{2 \pi a t}-1}\right] .
$$

Using Eqs.(55) and (57) in Eq.(54), we get a polar part (size independent) plus a size dependent analytic correction. It is clear that the mass counterterm generated by $f_{1}(L, m, d)$ is size independent, as the finite temperature field theory has no temperature dependent counterterm. The first interesting result of the paper is given by Eqs.(51) and and (53). The tadpole graphs expressed by $T_{D D}(L, m, d, z)$ and $T_{N N}(L, m, d, z)$ have the same $z$ dependent part in modulus but with opposite signs. From the above discussion it is possible to understand the finiteness of the renormalized stress-tensor of an electromagnetic field near a flat prefectly conducting plate. Although the expectation value of the squared electric and magnetic field are divergent, a delicate cancellation makes the renormalized stress-tensor finite. As the size-dependent parts of $T_{D D}(L, m, d, z)$ and 
$T_{N N}(L, m, d, z)$ have the same functional form and opposite signs, and recalling that it is possible to treat the electric and magnetic modes separately (where one obeys $D D$ and the other $N N$ b.c., we automatically obtain a finite result for the vacuum expectation value of the stress-tensor of the electromagnetic field. It is important to stress that when the conducting boundary is curved, the energy density diverges on the boundary [29].

To shall deal with the renormalization program in the one-loop approximation in the next section, also discussing, for the sake of completeness, the issue of IR divergencies in different numbers of dimensions.

\section{Analysis of the ultraviolet and infrared divergences of the $z$-dependent part of the tadpoles}

We will again use the fact that $d^{d-1} p=p^{d-2} d p d \Omega_{d-1}$ and $\int d \Omega_{d-1}=\frac{2 \frac{d-1}{2}}{\Gamma\left(\frac{d-1}{2}\right)}$. It should be noted that, had we chosen $m^{2}=0$, the ultraviolet divergences would have kept the same polar structure. Consequently, for simplicity let us choose again $m=0$, and for reasons that will become evident latter, we first assume $d>3$. The special case $d=3$ is discussed at the end of this section. Defining $h_{2}(d)$ by:

$$
h_{2}(d)=\frac{1}{2^{d-2}} \frac{1}{\pi^{\frac{d-1}{2}}} \frac{1}{\Gamma\left(\frac{d-1}{2}\right)},
$$

it is possible to write $\left.f_{2}(L, m, d, z)\right|_{m=0}$ as

$$
\left.f_{2}(L, m, d, z)\right|_{m=0}=\frac{1}{2} h_{2}(d) \int_{0}^{\infty} d k k^{d-3} \operatorname{coth} k L \cosh 2 k z
$$




$$
\text { - } h_{2}(d) \int_{0}^{\infty} d k k^{d-3} \cosh k z \sinh k z
$$

In a general way, the regularization process is achieved introducing exponential cut-off regulators and after this the identification of the poles of the regularized quantity by means of the Laurent series expansion around some point i.e. the negative power portion of such series. Note that instead of imposing renormalization conditions over the 1PI correlation functions we can simply subtract the singular part of the Laurent series around some point, by the introduction of the counterterms. Let us assume $z \neq 0$ and $z \neq L$. A straightforward calculation gives

$$
\begin{aligned}
\left.f_{2}(L, m, d, z)\right|_{m=0} & =\frac{1}{2} h_{2}(d)\left[\int_{0}^{\infty} d k k^{d-3}(\operatorname{coth} k L-1) \cosh 2 k z\right. \\
& \left.+\int_{0}^{\infty} d k k^{d-3}(\cosh 2 k z-\sinh 2 k z)\right] .
\end{aligned}
$$

In the first integral for large $z,(\operatorname{coth} k L-1)$ has the behavior: $(\operatorname{coth} k L-1) \sim e^{-2 k L}$. Moreover, the second integral in the above equation is ultraviolet finite for $z \neq 0$. Let us define $x=k L$ and $q=k z$ in the first and second integrals above respectively. Then Eq.(60) becomes:

$$
\begin{aligned}
\left.f_{2}(L, m, d, z)\right|_{m=0} & =\frac{1}{2} h_{2}(d) \frac{1}{L^{d-2}} \int_{0}^{\infty} d x x^{d-3}(\operatorname{coth} x-1) \cosh \left(\frac{2 z}{L} x\right) \\
& +\frac{1}{2} h_{2}(d) \frac{1}{z^{d-2}} \int_{0}^{\infty} d q q^{d-3}(\cosh 2 q-\sinh 2 q) .
\end{aligned}
$$

The second term in the above equation gives us the well known result that for a massless minimal coupled scalar field $\left\langle\varphi^{2}(x)\right\rangle$ diverges as $\frac{1}{z^{2}}$ if we approach the plate [30. In order to analyze the polar part of $f_{2}(L, 0, d, z)$, we use the definition of the Gamma function. Let us define $I_{1}(\nu, \mu)$ 
and $I_{2}(\mu, \beta)$ by

$$
I_{1}(\mu, \nu)=\int_{0}^{\infty} d x x^{\mu-1} e^{-\nu x}=\frac{1}{\nu^{\mu}} \Gamma(\mu), \quad \operatorname{Re}(\mu)>0, \quad \operatorname{Re}(\nu)>0
$$

and

$$
I_{2}(\mu, \beta)=\int_{0}^{\infty} d x x^{\mu-1} e^{-\beta x}(\operatorname{coth} x-1)=2^{1-\mu} \Gamma(\mu) \zeta\left(\mu, \frac{\beta}{2}+1\right) \quad \operatorname{Re}(\beta)>0, \quad \operatorname{Re}(\mu)>1
$$

where $\zeta(z, a)$ is the Riemman zeta function defined by 24]

$$
\zeta(z, a)=\sum_{n=0}^{\infty} \frac{1}{(n+a)^{z}}, \quad \operatorname{Re}(z)>1, \quad a \neq 0,-1,-2 \ldots
$$

Then, using Eqs. (62), (63) and (64) in Eq. (61) we have that:

$$
\begin{aligned}
\left.f_{2}(L, m, d, z)\right|_{m=0} & =\frac{1}{2} h_{2}(d) \frac{1}{L^{d-2}}\left[2^{2-d} \Gamma(d-2)\left(\zeta\left(d-2, \frac{z}{L}+1\right)+\zeta\left(d-2,-\frac{z}{L}+1\right)\right)\right] \\
& +\frac{1}{(2 z)^{d-2}} h_{2}(d) \Gamma(d-2) .
\end{aligned}
$$

Using the definition of the zeta function, it is evident that:

$$
\begin{aligned}
& \frac{1}{L^{d-2}}\left(\zeta\left(d-2, \frac{z}{L}+1\right)+\zeta\left(d-2,-\frac{z}{L}+1\right)\right)= \\
& \frac{1}{L^{d-2}} \sum_{n=0}^{\infty} \frac{1}{\left(n+\left(1+\frac{z}{L}\right)\right)^{d-2}}+\frac{1}{(L-z)^{d-2}}+\frac{1}{L^{d-2}} \sum_{n=1}^{\infty} \frac{1}{\left(n+\left(1-\frac{z}{L}\right)\right)^{d-2}} .
\end{aligned}
$$

We see that the regularized $f_{2}(L, 0, d, z)$ has two poles of order $(d-2)$ in $z=0$ and in $z=L$. Note that the residues of the poles in $z=0$ and in $z=L$ are $L$-independent. Since the domain of analyticity of the zeta function is $d>3$, the case $d=3$ must be studied separately. Different treatments for $d=3$ and $d=4$ simply express the fact that infrared divergences are more severe in lower dimensions. 
We will go back to Eq.(52), studying the case $m^{2} \neq 0$, to see how the IR divergences pop up in the $m^{2} \rightarrow 0$ limit. It is important to stress that, only in the N-N B.C.'s case we have IR divergences for massless fields, coming from the term $n=0$, i.e., equations (51) and (52) are IR finite for $m=0$. A straightforward calculation yields

$$
f_{2}(L, m, d, z)=\frac{1}{2} h_{2}(d) \int_{0}^{\infty} d \rho \frac{\rho^{d-2}}{\left(\rho^{2}+m^{2}\right)^{\frac{1}{2}}} \frac{\cosh \left((L-2 z)\left(\rho^{2}+m^{2}\right)^{\frac{1}{2}}\right)}{\sinh \left(L\left(\rho^{2}+m^{2}\right)^{\frac{1}{2}}\right)} .
$$

Defining $\sigma=\left(\rho^{2}+m^{2}\right)^{\frac{1}{2}}$ and using the fact that $d=3$, we have:

$$
\begin{aligned}
\left.f_{2}(L, m, d, z)\right|_{d=3} & =\frac{1}{2} h_{2}(3)\left[\int_{m}^{\infty} d \sigma(\operatorname{coth} \sigma L-1) \cosh 2 \sigma z\right. \\
& \left.+\int_{m}^{\infty} d \sigma(\cosh 2 \sigma z-\sinh 2 \sigma z)\right] .
\end{aligned}
$$

The second integral in the above expression is convergent for $z \neq 0$, and defining $v=2 \sigma z$, it becomes:

$$
\frac{1}{4 z} h_{2}(3) \int_{2 m z}^{\infty} d v e^{-v}=\frac{1}{4 z} h_{2}(3) \Gamma(1,2 m z),
$$

where $\Gamma(a, x)$ is the incomplete gamma function. Consequently, we have a simple pole for $z=0$. Again, the residue of this pole is $L$-independent. To complete the regularization procedure we have now to analyze the first integral of Eq. (68):

$$
\frac{1}{2} h_{2}(3) \int_{m}^{\infty} d \sigma(\operatorname{coth} \sigma L-1) \cosh 2 \sigma z=\frac{1}{4 L} h_{2}(3) \int_{m L}^{\infty} d u \frac{e^{\frac{z}{L} u}}{e^{u}-1}+\frac{1}{4 L} h_{2}(3) \int_{m L}^{\infty} d u \frac{e^{-\frac{z}{L} u}}{e^{u}-1} .
$$

The second integral in the right side of Eq.(70) is convergent and the first one has a simple pole at $z=L$, again with an $L$-independent residue. 
From the discussion above, we can conclude that, in order to eliminate the ultraviolet divergences of the theory we have to introduce counterterms as surface interactions, and consequently the full action will have the following form for both fields $\varphi_{1}$ and $\varphi_{2}$ :

$$
S(\varphi)=\int_{0}^{L} d z \int d^{d-1} r\left(\frac{1}{2}(\partial \varphi)^{2}+\frac{1}{2} m^{2} \varphi^{2}+\frac{1}{4 !} \lambda \varphi^{4}\right)+\int d^{d-1} r\left(c_{1} \varphi^{2}(\vec{r}, 0)+c_{2} \varphi^{2}(\vec{r}, L)\right) .
$$

As we have already taken care of the ultraviolet divergences, let us study the infrared divergent piece (for $m=0$ ) of $\left.f_{2}(L, m, d, z)\right|_{d=3}$. Let us call this piece $\left.f_{2}^{*}(L, m, d, z)\right|_{d=3}$. Note that we introduce an ultraviolet cut-off in order to use the Bernoulli representation of the integrand.

$$
\left.f_{2}^{*}(L, m, d, z)\right|_{d=3}=\frac{1}{4 L} h_{2}(3)\left(\int_{m L}^{2 \pi} d u \frac{e^{\frac{z}{L} u}}{e^{u}-1}+\int_{m L}^{2 \pi} d u \frac{e^{-\frac{z}{L} u}}{e^{u}-1}\right) .
$$

Writing the integrand using the Bernoulli polynomials it is not difficult to show that

$$
f_{2}^{*}(L, m, 3, z)=\frac{1}{2 L} h_{2}(3) B_{0}\left(\frac{z}{L}\right) \ln \left(\frac{2 \pi}{m L}\right)+\text { regular part }\left(f_{2}^{*}(L, m, z)\right) .
$$

When $m \rightarrow 0$ we have a logarithmic divergence which is $z$ dependent.

Going back to the case of the ultraviolet divergence, some authors claimed that the introduction of surface counterterms is against the spirit of the renormalization program. In our case, however, it is possible to change the model, by adding a new interaction term, in such a way that the ultraviolet divergences coming from the $f_{2}(L, m, d, z)$ contributions corresponding to each field are compensated. One possibility is to consider the $O(2)$ symmetric model with a $\varphi_{1}^{2} \varphi_{2}^{2}$ interaction term. Then the $z$-dependent part of each tadpole cancel each other out. The situation is similar to the case of supersymmetric theories, where the finiteness of some correlation functions is achieved 
by a balance between bosonic and fermionic loops. The $O(2)$ symmetric model with an interaction term $\varphi_{1}^{2} \varphi_{2}^{2}$ (DD and $N N$ B.C.'s) developes a size dependent mass $\Delta m^{2}$ proportional to $g L^{-2}$, as the $\varphi^{4}$ model at finite temperature. In the same way as temperature can solve the IR problem in some QFT models, finite size effects can also cure these divergences, when a resummation can be implemented.

As stressed by many authors, in the electromagnetic case, the origin of the unboundedness renormalized stress-tensor near a curved surface has the origin in the unphysical nature of classical "perfect conductor" boundary condition. Let us suppose the following physical situation. For the low energy modes the manifold is $[0, L] \times \Re^{d-1}$ and for the high energy modes we have $S^{1} \times \Re^{d-1}$, i.e. let us assume a sharp cut-off and for $k_{n}<\Lambda$ we have $D D$ boundary conditions and for $k_{n} \geq \Lambda$ we have periodic B.C.'s. The high frequencies do not "see" the mirror at $z=0$, and translational invariance is maintained only for these modes. If the collapse of the renormalization program (removing infinities from perturbative calculations using only "bulk" counterterms) is related with the break of translational invariance, our improved model must be renormalizable. A further study of this model may be of interest. A different possibility is to construct an effective action for the slow-modes and after this imposing the $D D$ or $N N$ b.c. 31 32.

\section{Conclusions}

In this paper we studied finite size effects in an interacting field theory, with broken translation invariance. We calculated the vacuum activity for an anisotropic model, between two parallel plates 
in a $d$ dimensional Euclidean space. It has been possible to obtain closed expressions for $\left\langle\varphi_{1}^{2}(x)\right\rangle$ and $\left\langle\varphi_{2}^{2}(x)>\right.$, for fields satisfying $D D$ and $N N$ B.C.'s, respectively. For different shapes, the complexity and the number of technical difficulties increase enormously, but the multiple scattering method can be used in these cases [1], at least for small curvatures of the boundaries. We presented a model having the interesting property that the $z$-dependent part of the tadpole graphs for $D D$ and $N N$ B.C.'s have the same modulus and opposite signs. This fact could explain the boundedness of the renormalized vacuum expectation value of the energy-stress tensor of the electromagnetic field in the Casimir-like configuration.

We have also seen that, to renormalize the theory, counterterms corresponding to surface interactions are required. One can, however, avoid this difficulty by equipping the model with a $\varphi_{1}^{2} \varphi_{2}^{2}$ interaction. Then, the $z$ dependent pieces of each tadpole cancel each other out, and the two fields develop a size dependent mass $\Delta m^{2}$ proportional to $g L^{-2}$, as for the single $\varphi^{4}$ model at finite temperature.

There are several directions in which the finite size effects for systems with breaking of translational invariance which may deserve further research. To mention a few of them: the study of interacting fermions, the non-linear $\sigma$ model in domains with one finite direction and $(d-1)$ infinite directions [33], and finally as a straightforward extension of this work, the study of the $O(2)$ symmetric model at the two-loop approximation.

As discussed in the Introduction, one should prove that the the renormalization program can be implemented beyond the one-loop approximation, where overlapping divergences emerge. 


\section{Acknowlegements}

We would like to thank B.Schroer and R.De Paola for several helpful discussions. We are also grateful to B.F.Svaiter for very useful discussions and comments. N.F.Svaiter would like to acknowledge the hospitality of the Centro Atómico Bariloche where part of this work was carried

out.This paper was supported by Conselho Nacional de Desenvolvimento Cientifico e Tecnologico do Brazil (CNPq) and Centro Latino Americano de Fisica (CLAF).

\section{References}

[1] R.Balian and C.Bloch, Ann.Phys. 60, 401 (1970), R.Balian and B.Duplantier, Ann.Phys.112, 165 (1978).

[2] A.A.Actor and I.Bender, Phys.Rev.D 52, 3581 (1995); L.C.Albuquerque, Phys.Rev.D 55, 7752 (1997); F.Caruso, R.De Paola and N.F.Svaiter, Int.Journ.Mod.Phys.A14, 2077 (1999).

[3] L.H.Ford and N.F.Svaiter, Phys.Rev. D 58, 065007-1 (1998).

[4] C.S.Zasada and and P.K.Pathria, Phys. Rev.A 14, 1269 (1976); S.Singh and P.K.Pathria, Phys.Rev.A 30, 442 (1984).

[5] E.Brezin and J.Zinn-Justin, Nucl.Phys.B257, 867 (1985).

[6] A.N.Nemirovsky and K.F.Freed, J.Phys.A 18, L319 (1985). 
[7] S.Singh and P.K.Pathria, Phys. Rev.B45, 9759 (1992).

[8] H.D.Diehl and S. Dietrich, Phys.Rev.B 24, 2878 (1981); H.D.Diehl and S. Dietrich, Phys.Lett. 80A, 408 (1981); Y.Y. Goldschmit, Phys.Rev.B 28, 4052 (1983); A.N.Nemirovsky and K.F.Freed, Phys.Rev.B 31, 3161 (1985).

[9] L.H.Ford and M.Yoshimura, Phys.Lett 70A, 89 (1979); D.J.Toms, Phys.Rev.D 21, 928 (1980); D.J.Toms, Phys. Rev.D 21, 928 (1980); G.Denardo and E.Spalucci, Nucl.Phys. B169, 514 (1980); Y.P.Goncharov, Phys.Lett 91A, 153 (1982); Y.Hosotani, Phys.Lett. 126B, 309 (1983).

[10] A.D.Linde, Rep.Prog.Phys.42, 390 (1979); D.A. Kirzhnitz and A.D.Linde, Ann. of Phys. 101, 195 (1976); L.Dolan and R.Jackiw, Phys. Rev D 9, 3320 (1974).

[11] L.H.Ford and N.F.Svaiter, Phys.Rev. D 51, 6981 (1995).

[12] A.Chodos, R.L.Jaffe, C.B.Thorn and V.F.Weisskopf, Phys.Rev.D 9, 3471 (1974), T.H.Hansson and K.Johnson and C.Peterson, Phys.Rev.D 26, 915 (1982); T.H.Hanson and R.L.Jaffe, Phys.Rev. D 28, 882 (1983), T.H.Hanson and R.L.Jaffe, Ann.Phys. 151, 204 (1983).

[13] M.Bordag, D.Robaschik and E.Wieczorek, Ann.Phys. 165, 192 (1985), D.Robaschik, K.Schrnhorst and E.Wieczorek, Ann.Phys. 174, 401 (1987); Chodos and C.B.Thorn, Phys.Lett. 53B, 359 (1974). 
[14] D.J.Toms, Ann.of Phys. 129, 334 (1980).

[15] N.D.Birrel and L.H.Ford, Phys.Rev D 22, 330 (1980).

[16] R.Banach, J.Phys.A13, 1365 (1980).

[17] J.C.Taylor, Phys.Rev.84, 897 (1951), J.G.Taylor, Nuovo Cimento Supp.1, 857 (1963).

[18] K.Zymanzik, Nucl.Phys.B 190, 1, (1980).

[19] A.M.Nemirovsky and K.F.Freed, Nucl.Phys.B 270, 423, (1986), M.Krech and S.Dietrich, Phys.Rev.A 46, 1886 (1992).

[20] J.F.Ashmore Nuovo Cim.Lett. 9, 289 (1972), C.G.Bolini and J.J.Giambiagi Nuovo Cim.B 12, 20 (1972), G.t'Hooft and M.Veltman Nucl.Phys.B 44, 189 (1972), G.Leibrant, Rev.Mod.Phys. 47, 849 (1975).

[21] W.Lukosz, Z.Phys.258, 99 (1973), G.Plunien, B.Muller and W.Greiner, Phys.Rep. 134, 87 (1986).

[22] C.D.Fosco and A.Lopez, Nuc.Phys.B 538, 685 (1999).

[23] H.W.Diehl and S.Dietrich, Z.Phys. B42, 65 (1981).

[24] I.S.Gradshteyn and I.M.Ryzhik, Tables of Integrals, Series and Products (Academic Press Inc., New York 1980) 
[25] J.I.Kapusta, Finite Temperature Field Theory (Cambridge U.P. New York 1989), N.P.Landsman and C.G.Van Weert, Phys. Rep. 145, 141 (1987), N.Weiss, Phys.Rev.D27, 899 (1983).

[26] S.Lang in Complex Analysis, Springer Verlag, New York (1993).

[27] A.P.C.Malbouisson and N.F.Svaiter, Physica A 233, 573 (1996); ibid J.Math.Phys. 37, 4352 (1996).

[28] L.H.Ford, Phys.Rev.D 21, 933 (1980).

[29] D.Deutsch and P.Candelas, Phys.Rev. D 20, 3063 (1979); P.Candelas, Ann.Phys. 144, 241 (1982); P.C.W.Davies and D.J.Toms, Phys.Rev.D 31, 1363 (1985).

[30] S.A.Fulling, Aspects of Quantum Field Theory on Curved Spacetime, Cambridge University Press, Cambridge (1989).

[31] Wilson, K.G. and J.B.Kogut, Phys.Rep. 12, 7 (1974); J.Polchinski, Nucl.Phys. 231, 269 (1984), S.Ben Liao and J.Polonyi, Ann.Phys. 222, 122 (1993); R.Shankar, Rev.Mod.Phys. 66, $129(1994)$

[32] K.Scharnhorst, Phys.Lett.B 236, 354 (1990); G.Barton, Phys.Lett.B 237, 559 (1990); D.Hennig and D.Robaschik, Phys.Lett.A 151, 209 (1990); M.Bordag, D.Hennig and D.Robaschik, J.Phys.A 25, 4483 (1992); G.Barton and K.Scharnhorst, J.Phys.A 26, 2037 (1993); X.Kong and F.Ravndal, Phys.Rev.Lett. 79, 545 (1997). 
[33] E.G.Floratos and D.Petcher, Nucl.Phys. 252, 689 (1985). 